\title{
Liver hemojuvelin protein levels in mice deficient in matriptase-2 (Tmprss6)
}

\author{
Jan Krijt ${ }^{\text {a, } *, 1}$, Yuzo Fujikura ${ }^{\text {a, }}{ }^{,}$, Andrew J. Ramsay ${ }^{\text {b }}$, Gloria Velasco ${ }^{\text {b }}$, Emanuel Nečas ${ }^{\text {a }}$ \\ a Institute of Pathophysiology and Center of Experimental Hematology, Charles University in Prague, First Faculty of Medicine, U Nemocnice 5 , 128 53 Prague, Czech Republic \\ ${ }^{\mathrm{b}}$ Departamento de Bioquímica y Biología Molecular, Facultad de Medicina, Instituto Universitario de Oncología, Universidad de Oviedo, Julian Claveria s/n, 33006 Oviedo, Spain
}

\section{A R T I C L E I N F O}

\section{Article history:}

Submitted 3 November 2010

Revised 18 March 2011

Available online 25 May 2011

Communicated by A. Townsend, MD, PhD, FRCP, FRS, 19 April 2011

\section{Keywords:}

Iron metabolism

Hepcidin

Hemojuvelin

TMPRSS6

\begin{abstract}
A B S T R A C T
Mutations of the TMPRSS6 gene, encoding the serine protease matriptase-2, lead to iron-refractory iron deficiency anemia. Matriptase-2 is a potent negative regulator of hepcidin. Based on in vitro data, it has recently been proposed that matriptase- 2 decreases hepcidin synthesis by cleaving membrane hemojuvelin, a key protein of the hepcidin-regulatory pathway. However, in vivo evidence for this mechanism of action of matriptase-2 is lacking. To investigate the hemojuvelin-matriptase-2 interaction in vivo, an immunoblot assay for liver membrane hemojuvelin was optimized using hemojuvelin-mutant mice as a negative control. In wild-type mice, two hemojuvelin-specific bands of $35 \mathrm{kDa}$ and $20 \mathrm{kDa}$ were detected in mouse liver membrane fraction under reducing conditions; under non-reducing conditions, a single band of approximately $50 \mathrm{kDa}$ was seen. Phosphatidylinositol-specific phospholipase $\mathrm{C}$ treatment confirmed binding of the detected protein to the cell membrane by a glycosylphosphatidylinositol anchor, indicating that the major form of mouse liver membrane hemojuvelin is a glycosylphosphatidylinositol-bound heterodimer. Unexpectedly, comparison of liver homogenates from Tmprss6 $+1+$ and Tmprss6-1- mice revealed significantly decreased, rather than increased, hemojuvelin heterodimer content in Tmprss6-/- mice. These data do not provide direct support for the concept that matriptase- 2 cleaves membrane hemojuvelin and may indicate that, in vivo, the role of matriptase- 2 in the regulation of hepcidin gene expression is more complex.
\end{abstract}

(c) 2011 Elsevier Inc. All rights reserved.

\section{Introduction}

Iron homeostasis in man is precisely regulated at the level of iron absorption in the small intestine. The key iron metabolism regulatory compound is hepcidin, a hepatocyte-derived peptide which controls iron export from macrophages and enterocytes [1,2]. Hepcidin expression is in turn determined by several signaling pathways, which modulate hepcidin transcription in response to iron stores, inflammation and the rate of erythropoiesis [3]. The main component of the iron-dependent regulation is hemojuvelin ( $\mathrm{Hjv})$, a hepatocyte membrane protein encoded by the HFE2 gene. Hjv interacts with bone morphogenetic protein ligands [4], and this interaction at the hepatocyte membrane initiates a cascade of intracellular phosphorylations which ultimately lead to an increase in hepcidin gene transcription. The importance of $\mathrm{Hjv}$ in hepcidin regulation is evident from the fact that mutations in the HFE2 gene cause severe juvenile hemochromatosis, which has similar phenotype as juvenile hemochromatosis caused by mutations in the hepcidin gene itself [5].

\footnotetext{
* Corresponding author at: Institute of Pathophysiology, Charles University in Prague, First Faculty of Medicine, U Nemocnice 5, 12853 Prague, Czech Republic. Fax: + 420224912834

E-mail address: jkri@lf1.cuni.cz (J. Krijt).

1 These authors contributed equally to the study.
}

Recently, another membrane protein participating in the control of iron metabolism has been identified. The serine protease matriptase2 , encoded by the TMPRSS6 gene, is a negative regulator of hepcidin expression [6-8]. TMPRSS6 mutations cause inappropriately high expression of hepcidin, resulting in diminished iron absorption, iron sequestration in macrophages, and, ultimately, iron-refractory iron deficiency anemia. It has been demonstrated that, under in vitro conditions, matriptase-2 efficiently cleaves membrane hemojuvelin $[9,10]$, and similar mechanism of hemojuvelin protein regulation is postulated to function in vivo. According to the current model, mutated forms of matriptase- 2 would be unable to cleave membrane $\mathrm{Hjv}$, resulting in the presence of high levels of Hjv on the hepatocyte membrane, continued stimulation of the bone morphogenetic protein signaling pathway, and inappropriately high hepcidin expression. The model has been recently further strengthened by reports that mice deficient in both Hjv and matriptase-2 display the same low hepcidin mRNA levels as mice deficient in Hjv [11,12].

Despite the importance of hemojuvelin in iron metabolism, there are only few experimental studies dealing with Hjv protein levels in vivo. The main reason for this absence of experimental in vivo data is the unavailability of robust antibodies that recognize endogenous murine hemojuvelin in tissue lysates [11,13]. Commercial antibodies display non-specific bands [13], whose presence makes the identification of Hjv protein on immunoblots unreliable. Therefore, the aim of the present study was to develop a reliable Hjv immunoblot assay, by 
using $\mathrm{Hjv}-/-$ mice as a negative control. As a next step, the optimized assay was employed to compare membrane Hjv protein levels between Tmprss6+1+ and Tmprss6-/- mice. Unexpectedly, it was found that the content of liver membrane-bound Hjv heterodimer, the major form of Hjv detected by the assay, was decreased, rather than increased, in mice lacking matriptase-2.

\section{Methods}

Hjv-/- mice [14] were a generous gift from Prof. Silvia Arber, Basel, Switzerland. Generation and biochemical characterization of Tmprss6-/ - mice have been described previously [8], the animals display increased Hamp mRNA levels and absent Tmprss6 expression. Male Tmprss6-/- and Tmprss6 $+1+$ mice, aged 8 weeks, were used for Hjv determinations. All animal experiments were approved by the Ethics Committee of the First Faculty of Medicine in Prague. Anti-Hjv antibody (AF3634), generated against the mature region of mouse Hjv (amino acids 36-393), was purchased from R\&D Systems (Minneapolis, MN, USA), anti-pan-cadherin antibody (4068) from Cell Signaling (Boston, MA, USA). Secondary antibodies (705-036147 and 713-036-137) were obtained from Jackson ImmunoResearch Europe, UK.

For Hjv protein determination in whole tissue lysates, tissue samples were homogenized $(5 \times 10 \mathrm{~s})$ with an Ultra Turrax homogenizer in 4 volumes of Ripa buffer (Sigma Aldrich) with a protease inhibitor mix (Roche Diagnostics, Mannheim, Germany) and centrifuged for $15 \mathrm{~min}$ at $6000 \times \mathrm{g}$. $80 \mu \mathrm{g}$ of supernatant protein was loaded on a pre-cast $4-20 \%$ Tris-Glycine minigel (Invitrogen, Carlsbad, CA, USA). For liver membrane Hjv protein determinations, liver was homogenized in 5 volumes of $0.25 \mathrm{mM}$ sucrose, $\mathrm{pH}$ 7.6, containing $1 \mathrm{mM}$ EDTA and protease inhibitors. Homogenate was centrifuged for $15 \mathrm{~min}$ at $6000 \times \mathrm{g}$, and membranes were obtained by ultracentrifugation of the supernatant at $80,000 \times \mathrm{g}$ for $45 \mathrm{~min}$. $60 \mu \mathrm{g}$ of the membrane protein was separated by SDS electrophoresis under both reducing ( $2 \% \beta$-mercaptoethanol) and non-reducing conditions. Proteins were blotted on a PVDF membrane, blots were blocked with $5 \%$ skimmed milk in tris-buffered saline containing $0.1 \%$ of Tween 20, and incubated overnight with a solution of the primary antibody in $5 \%$ milk. Primary antibody dilutions were $1: 1000$ for hemojuvelin, 1:100,000 for Gapdh and 1:4000 for pan-cadherin. After washing, blots were incubated with a $1: 20,000$ solution of the secondary antibody in 5\% milk and proteins were visualized by chemiluminescence. Densitometry was performed on a Biorad GS-80 scanner, statistical analyses of densitometry and PCR results were performed using the Mann-Whitney nonparametric test.

For phosphatidylinositol-specific phospholipase C (Pi-PLC) cleavage of the GPI anchor, liver membrane samples (approximately $2 \mathrm{mg}$ of protein) were treated with Pi-PLC (Sigma-Aldrich, $100 \mathrm{mU}$ ) at $37^{\circ} \mathrm{C}$ for 3 hours and subsequently recentrifuged at $45,000 \times \mathrm{g}$ for $45 \mathrm{~min}$. $60 \mu \mathrm{g}$ of supernatant protein was loaded on gel for immunoblots.

Liver mRNA isolation was performed as previously described [8]. For real-time PCR determination of hepcidin gene (Hamp) and Hjv gene (Hfe2) expression, a Roche LightCycler instrument in combination with the Fast Start SYBR Green protocol (Roche LightCycler, Roche Diagnostics GmBH, Germany) was used. Target mRNA content was calculated relatively to Gapdh mRNA content, assuming exact doubling of amplified DNA in each PCR cycle. Primer sequences (forward and reverse) were Gapdh, CGGTGTGAACGGATTTGC and GCAGTGATGGCATGGACTGT; Hfe2, CAATCCTGCGTCTTTGATGTT and GAAGCAAAGCCACAGAACAAA; Hamp CTGAGCAGCACCACCTATCTC and TGGCTCTAGGCTATGTTTTGC.

\section{Results}

Immunochemical detection of $\mathrm{Hjv}$ in whole liver homogenates resulted in several non-specific bands; however, comparison of samples from $H j v+/+$ and $H j v-/$ - mice enabled clear identification of two hemojuvelin-specific bands at approximately $35 \mathrm{kDa}$ and $20 \mathrm{kDa}$ (Fig. 1A). The $35 \mathrm{kDa}$ band appeared more strongly on immunoblots than the $20 \mathrm{kDa}$ band and was therefore used for subsequent densitometry quantifications. Hemojuvelin-specific bands were also seen in homogenates of muscle and myocardium (Supplementary Figure 1). This tissue distribution of hemojuvelin protein is in agreement with the tissue distribution of Hfe 2 mRNA, which is present in mainly muscle, myocardium and liver [5]. In contrast to previously reported data [15], no Hjv-specific bands were detected in homogenates from kidney or testis (Supplementary Figure 1).

Hjv protein exists in soluble and membrane-bound forms [16]. Since signal transduction by the Hjv/Smad pathway depends on membrane-bound hemojuvelin, crude membrane fractions were utilized for Hjv determination in subsequent experiments. Membranes were prepared by ultracentrifugation, and membrane Hjv content was examined in livers from $\mathrm{Hjv}+\mathrm{I}+\mathrm{Hjv}+\mathrm{I}-$ and $\mathrm{Hjv}-\mathrm{I}-$ mice. The results again showed the presence of the 35 and $20 \mathrm{kDa}$ bands in the liver membrane fraction of $\mathrm{Hjv}+/+$ and $\mathrm{Hjv}+/-$ mice (Fig. 2A); compared to $\mathrm{Hjv}+/+$ mice, the intensity of the $35 \mathrm{kDa}$ band was decreased in $\mathrm{Hjv}+/-$ mice (Fig. 2B). Levels of $\mathrm{Hfe} 2 \mathrm{mRNA}$ were decreased in $\mathrm{Hjv}+/$ - mice by about 50\% (Fig. 3A). As noted previously [17], there was no significant change in Hamp mRNA levels between $\mathrm{Hjv}+/$ - and $\mathrm{Hjv}+$ / + mice (Fig. 3B).

At present, it is not exactly known which forms of Hjv are expressed at the mouse hepatocyte membrane in vivo. One of the possible candidates is a glycosylphosphatidylinositol (GPI) anchored heterodimer, composed of two disulfide bond-linked Hjv fragments (AA 33-165 and AA 166-393), which originate by autoproteolytic cleavage of the full-length peptide at a labile Asp-Pro bond [16,18,19]. The observed size of Hjv-specific bands obtained under reducing conditions (Fig. 4A) agrees with this concept, provided the fragments are glycosylated as predicted $[16,18,19]$. When liver membranes were analyzed under non-reducing conditions, the Hjv-specific bands moved as a single spot of approximately $50 \mathrm{kDa}$ (Fig. 4B), which is again in agreement with the reported heterodimer composition. Hjvspecific bands were found only in membrane fraction; following ultracentrifugation of liver homogenate, no Hjv-specific bands were seen in the supernatant (Fig. 4A). To confirm that the detected protein is indeed bound to the membrane by a GPI anchor, membrane samples were treated with Pi-PLC and recentrifuged. As expected for a GPI-bound protein, after Pi-PLC treatment the Hjv-specific bands were found in the supernatant fraction, both in reduced and non-reduced samples. In reduced samples, Pi-PLC treatment decreased the intensity of the $20 \mathrm{kDa}$ band; however, the signal of the major $35 \mathrm{kDa}$ band significantly increased, and a new minor Hjv-specific

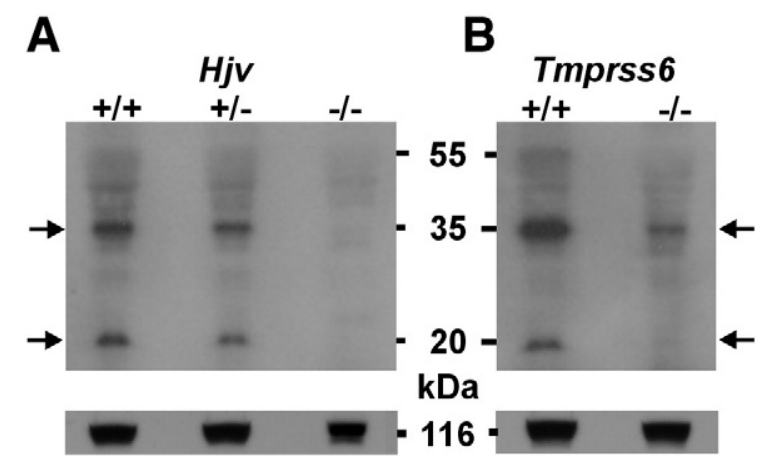

Fig. 1. Hemojuvelin protein in whole liver homogenates. A: Immunoblot of Hjv protein in whole liver homogenates from $\mathrm{Hjv}+/+, \mathrm{Hjv}+/-$ and $\mathrm{Hjv}-/-$ mice. B: Immunoblot of Hjv protein in whole liver homogenates from Tmprss6 $+1+$ and Tmprss6 $-/-$ mice. $80 \mu \mathrm{g}$ of protein was loaded per lane, pan-cadherin $(116 \mathrm{kDa})$ was used as loading control. Arrows indicate the positions of hemojuvelin-specific bands 
A

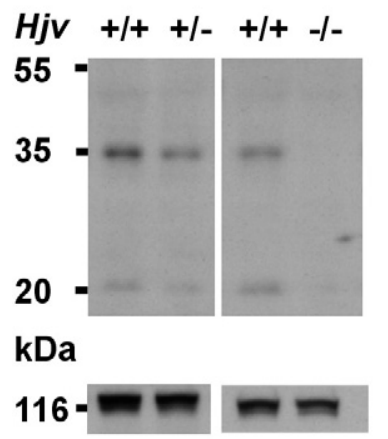

B

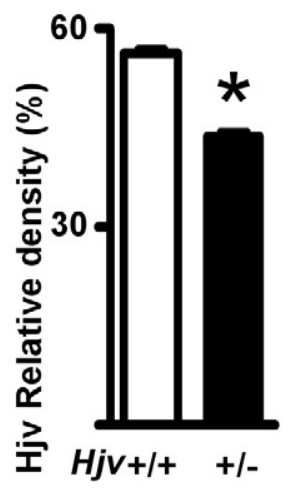

A

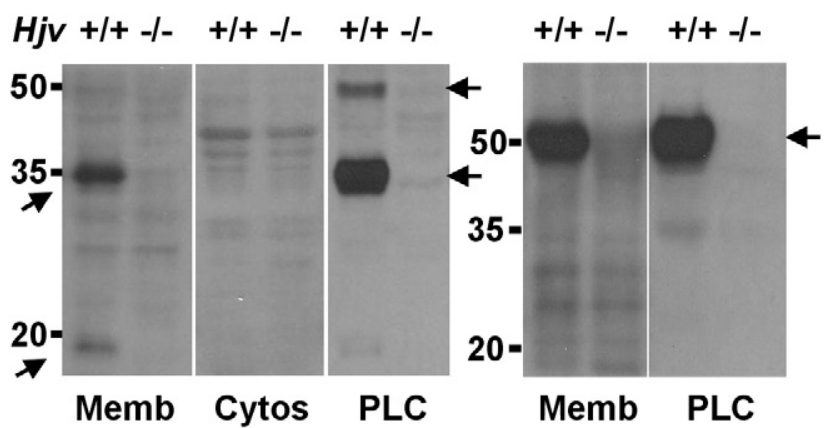

Fig. 2. Hemojuvelin protein in liver membrane fractions. A: Hjv protein in liver membrane fractions of $\mathrm{Hjv}+\mathrm{l}+, \mathrm{Hjv}+\mathrm{l}-$ and $\mathrm{Hjv}-\mathrm{l}-$ mice. B: Densitometry of the $35 \mathrm{kDa}$ band from immunoblots of $\mathrm{Hjv}+\mathrm{l}+$ and $\mathrm{Hjv}+/-$ mice. $60 \mu \mathrm{g}$ of protein was loaded per lane, pan-cadherin ( $116 \mathrm{kDa}$ ) was used as loading control. Asterisk denotes statistical significance $(p<0.05, n=5)$.

band of approximately $50 \mathrm{kDa}$ appeared on the immunoblots (Fig. 4A, lane 3 ). This band very probably represents the full-length GPI-bound hemojuvelin monomer.

As a next step, the effect of Tmprss6 gene disruption on hemojuvelin protein levels was examined. Preliminary comparison of whole liver homogenates from Tmprss $6+1+$ and Tmprss6 $-/-$ mice indicated reduced content of Hjv in Tmprss6-/- mice (Fig. 1B). This result was unexpected, since if the role of matriptase- 2 protein is to cleave membrane Hjv, then Hjv levels should be higher, rather than lower, in Tmprss6-/- mice. To exclude the possibility that disruption of the Tmprss6 gene affects hemojuvelin gene transcription, $\mathrm{Hfe} 2$ mRNA levels were determined by real-time PCR. Liver Hfe2 mRNA content was similar both in Tmprss6 $+1+$ and Tmprss6 $-1-$ mice (Fig. 3C).

Since matriptase-2 has been reported to cleave Hjv protein present at the cell membrane [9], the next set of experiments examined possible changes in membrane Hjv protein fraction. Comparison of liver membrane Hjv protein levels between Tmprss6-1- and Tmprss6+/+
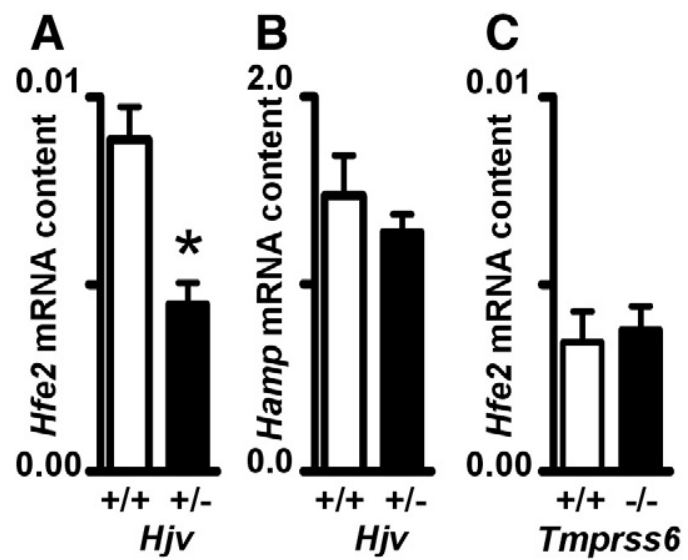

Fig. 3. Real-time PCR analysis of $H f e 2$ gene expression in livers of $H j v+/-$ and Tmprss6-/- mice. A: Hfe2 gene expression in livers of $\mathrm{Hjv}+/+$ and $\mathrm{Hjv}+/-$ mice. B: Hamp gene expression in livers of $\mathrm{Hjv}+/+$ and $\mathrm{Hjv}+/-$ mice. C: Hfe 2 gene expression in livers of Tmprss6+/+ and Tmprss6 $-/-$ mice. Target mRNA content is expressed relative to Gapdh mRNA, asterisk denotes statistical significance $(p<0.05), n=5$ for $\mathrm{Hjv}+/-$ mice and $n=3$ for Tmprss6 $-1-$ mice.

Fig. 4. GPI binding of liver membrane hemojuvelin. A: Samples from $\mathrm{Hjv}+/+$ and $\mathrm{Hjv}-/-$ mice run under reducing conditions. Memb: crude membrane fraction; Cytos: cytosol fraction (supernatant from liver homogenates, 80,000 $\times \mathrm{g}$, $45 \mathrm{~min}$ ); PLC: supernatant from samples obtained by treating the crude membrane fractions by phospholipase $C$ and subsequent recentrifugation. B: Samples from $\mathrm{Hjv}+/+$ and $\mathrm{Hjv}-/-$ mice run under nonreducing conditions. Memb: crude membrane fraction; PLC: supernatant from samples obtained by treating the crude membrane fractions by phospholipase $C$ and subsequent recentrifugation. $60 \mu \mathrm{g}$ of protein was loaded per lane, equal loading was verified by protein staining of the immunoblot membrane. Arrows indicate Hjv-specific bands.

mice again showed reduced content of the Hjv heterodimer in Tmprss6-/- animals, both under reducing (Fig. 5A) and nonreducing (Fig. 5B) conditions. Densitometry of the $35 \mathrm{kDa}$ (Fig. 5C) and $50 \mathrm{kDa}$ (Fig. 5D) protein bands confirmed the observations.
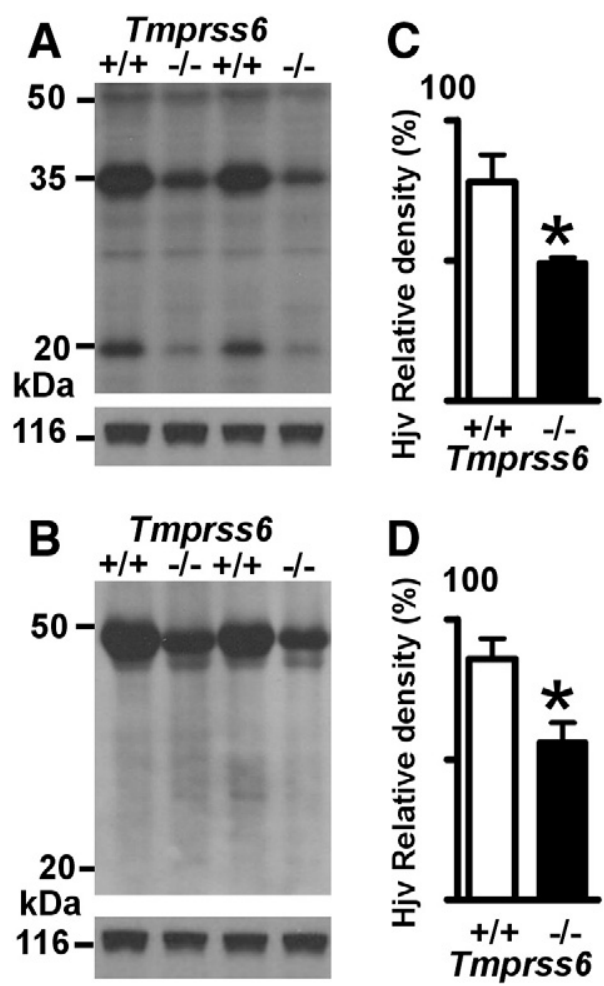

Fig. 5. Membrane hemojuvelin content in Tmprss6 - / - mice. A: Immunoblots of Hjv in liver crude membrane fractions from Tmprss $6+1+$ and Tmprss6 $-/-$ mice run under reducing conditions. B: Immunoblots of Hjv in liver crude membrane fractions from Tmprss6 + / + and Tmprss6-/- mice run under non-reducing conditions. Pan-cadherin $(116 \mathrm{kDa})$ was used for loading controls. $60 \mu \mathrm{g}$ of protein was loaded per lane. C: Quantification of densitometry data (35 kDa band) obtained from Tmprss6 $+1+$ and Tmprss6-/ - mice under reducing conditions $(n=4)$. D: Quantification of densitometry data (50 kDa band) obtained from Tmprss6 $+1+$ and Tmprss6 $-1-$ mice under nonreducing conditions $(n=3)$. Asterisks denote statistical significance $(p<0.05)$. 


\section{Discussion}

The purpose of the study was to optimize conditions for hemojuvelin protein detection, to obtain information about liver membrane hemojuvelin composition in vivo, and to determine hemojuvelin content in Tmprss6-/- mice.

Lack of reliable antibodies, which would detect hemojuvelin protein in whole tissue homogenates, is a well-known problem in hemojuvelin determinations [11,13]. However, when Hjv-/- mice were used as negative control, the optimized immunoblot procedure enabled identification of Hjv-specific bands both in whole liver homogenate and liver membrane fraction. The membrane-bound protein displayed the expected GPI binding; comparison of immunoblots run under reducing and non-reducing conditions agreed with the described heterodimer composition $[18,19]$. Based on these data, it appears that, in vivo, the major form of mouse liver membrane hemojuvelin is a GPI-bound heterodimer composed of two disulfidebound fragments, which originate by autoproteolytic cleavage of the full-length mature peptide [16].

An intriguing feature of the obtained results is the apparent lack of the expected $50 \mathrm{kDA}$ hemojuvelin monomer, which has been described in immunoblots of transfected cells [18-20], rat liver lysates [21] and rat hepatocytes [22], but which was seen in the present experiments only as a minor band in phospholipase-treated samples. Possibly, the $50 \mathrm{kDa}$ monomer could be less accessible to the antibody than the heterodimer; alternatively, the composition of hemojuvelin forms could be different between tissue homogenates and cultured cells. The predominance of the cleaved heterodimer in membrane fractions has been noted previously both in transfected cells [23] and mouse myoblast membranes [19].

Interestingly, the obtained data did not show the expected increase in liver hemojuvelin protein content in Tmprss6-/- mice. Thus, the results do not provide direct experimental support for the concept that matriptase- 2 regulates hepcidin transcription by cleaving membrane hemojuvelin. One possible explanation for this discrepancy with previously published studies $[9,10]$ is the fact that hemojuvelin cleavage by matriptase- 2 has been demonstrated in transfected cells in vitro, whereas the present study determines hemojuvelin content in mouse liver in vivo. While our data show a clear decrease of the GPI-bound heterodimer in whole liver homogenates and crude membrane fractions from Tmprss6-/animals, it is conceivable that matriptase- 2 actually cleaves some other, less abundant form of membrane hemojuvelin, which is not detected by the antibody used. A likely candidate could be the $50 \mathrm{kDa}$ hemojuvelin monomer. Alternatively, it is also possible that the actual amount of hemojuvelin protein at the plasma membrane represents only a small fraction of hemojuvelin present in the crude membrane preparation [24], which would make changes in plasma membrane hemojuvelin content difficult to detect. At present, it is not immediately apparent why the absence of matriptase-2 should result in decreased concentration of the hemojuvelin heterodimer; howev$\mathrm{er}$, it is evident that this decrease is not related to changes in Hfe2 gene transcription.

The reported interaction between matriptase- 2 and hemojuvelin $[9,10]$ suggests that, in vivo, matriptase- 2 regulates iron metabolism by cleaving membrane hemojuvelin, thus attenuating Bmp6-dependent signaling and ultimately decreasing transcription of the Hamp gene. However, it should be noted that there are no published data which would confirm that, under physiological conditions, liver Hamp mRNA content is actually regulated by changes of the amount of hemojuvelin protein. Rather, available information seems to indicate that Hamp mRNA levels are refractory to fluctuations in hemojuvelin protein content. For example, it has been reported that $\mathrm{Hjv}+\mathrm{/}-$ mice are, in terms of liver hepcidin expression or iron metabolism parameters, not different from $\mathrm{Hjv}+/+$ mice $[12,14,17]$. In this study, liver Hfe2 mRNA content in $\mathrm{Hjv}+\mathrm{/}-$ mice was, as expected, decreased to about $50 \%$ of the values found in $\mathrm{Hjv}+/+$ mice, and similar trend was observed for membrane Hjv protein levels. Nevertheless, Hamp mRNA content in $H j v+/-$ mice was not decreased, suggesting that hepcidin transcription proceeds unaffected even when membrane hemojuvelin content drops to one-half of normal. In addition, both $\mathrm{Hjv}+/+$ and $\mathrm{Hjv}+/-$ mice displayed similar increase in Hamp mRNA following iron overload (Supplementary Figure 2). Importantly, it has also been recently reported that mice overexpressing hemojuvelin do not display increased hepcidin mRNA levels, indicating that Hjv protein in hepatocytes is not a limiting factor for the modulation of hepcidin expression [25]. Taken together, these results do not suggest that hepcidin transcription would be sensitive to relatively small changes in membrane hemojuvelin content. Thus, in general terms, it can be stated that although the proposed physiological regulation of hepcidin expression by modulation of membrane hemojuvelin levels remains an attractive hypothesis, it still requires experimental validation. In addition, since the presented data do not confirm cleavage of membrane hemojuvelin by matriptase- 2 in vivo, the possibility that matriptase- 2 targets some other component of the hepcidin-regulatory pathway cannot be ruled out.

In conclusion, we developed an immunoblot assay for hemojuvelin protein determination in tissue samples and verified its validity by comparing samples from $\mathrm{Hjv}+/+$ and $\mathrm{Hjv}-/-$ mice. Using this assay, we found decreased liver hemojuvelin protein levels in Tmprss6-/mice. These results suggest that the role of matriptase- 2 in hepcidin gene regulation could be more complex than the well-documented in vitro cleavage of membrane hemojuvelin.

Supplementary materials related to this article can be found online at doi:10.1016/j.bcmd.2011.04.009.

\section{Acknowledgments}

Supported by grants MSM 0021620806, LC06044, and IGA MZCR NS10300-3/2009 (Czech Republic), and by funding from Ministerio de Ciencia e Innovación-Spain, Fundación M. Botín, and European Union (FP7 MicroEnviMet).

Contributions: J.K. and E.N. designed the study, J.K. and Y.F. performed research, J.K., Y.F. and A.J.R. analyzed data. A.J.R. and G.V. provided Tmprss6-/- mice. The authors thank Carlos-Lopez Otin, University of Oviedo, for data analysis and general help with the project.

The gift of $H j v-/-$ mice by Prof. Silvia Arber is gratefully acknowledged.

\section{References}

[1] E. Nemeth, T. Ganz, The role of hepcidin in iron metabolism, Acta Haematol. 122 (2009) 78-86.

[2] N.C. Andrews, Forging a field: the golden age of iron biology, Blood 112 (2008) 219-230

[3] M.W. Hentze, M.U. Muckenthaler, B. Galy, C. Camaschella, Two to tango: regulation of mammalian iron metabolism, Cell 142 (2010) 24-38.

[4] J.L. Babitt, F.W. Huang, D.M. Wrighting, Y. Xia, Y. Sidis, T.A. Samad, J.A. Campagna, R.T. Chung, A.L. Schneyer, C.J. Woolf, N.C. Andrews, H.Y. Lin, Bone morphogenetic protein signaling by hemojuvelin regulates hepcidin expression, Nat. Genet. 38 (2006) 531-539.

[5] G. Papanikolaou, M.E. Samuels, E.H. Ludwig, M.L. MacDonald, P.L. Franchini, M.P. Dubé, L. Andres, J. MacFarlane, N. Sakellaropoulos, M. Politou, E. Nemeth, J. Thompson, J.K. Risler, C. Zaborowska, R. Babakaiff, C.C. Radomski, T.D. Pape, O. Davidas, J. Christakis, P. Brissot, G. Lockitch, T. Ganz, M.R. Hayden, Y.P. Goldberg, Mutations in HFE2 cause iron overload in chromosome 1q-linked juvenile hemochromatosis, Nat. Genet. 36 (2004) 77-82.

[6] X. Du, E. She, T. Gelbart, J. Truksa, P. Lee, Y. Xia, K. Khovananth, S. Mudd, N. Mann, E.M. Moresco, E. Beutler, B. Beutler, The serine protease TMPRSS6 is required to sense iron deficiency, Science 320 (2008) 1088-1092.

[7] K.E. Finberg, M.M. Heeney, D.R. Campagna, Y. Aydinok, H.A. Pearson, K.R. Hartman, M.M. Mayo, S.M. Samuel, J.J. Strouse, K. Markianos, N.C. Andrews, M.D. Fleming, Mutations in TMPRSS6 cause iron-refractory iron deficiency anemia (IRIDA), Nat. Genet. 40 (2008) 569-571.

[8] A.R. Folgueras, F.M. de Lara, A.M. Pendás, C. Garabaya, F. Rodríguez, A. Astudillo, T. Bernal, R. Cabanillas, C. López-Otín, G. Velasco, Membrane-bound serine protease matriptase-2 (Tmprss6) is an essential regulator of iron homeostasis, Blood 112 (2008) 2539-2545 
[9] L. Silvestri, A. Pagani, A. Nai, I. De Domenico, J. Kaplan, C. Camaschella, The serine protease matriptase-2 (TMPRSS6) inhibits hepcidin activation by cleaving membrane hemojuvelin, Cell Metab. 8 (2008) 502-511.

[10] J. Maxson, J. Chen, C.A. Enns, A.S. Zhang, Matriptase-2 and proprotein convertasecleaved forms of hemojuvelin have different roles in the down-regulation of hepcidin expression, J. Biol. Chem. 285 (2010) 39021-39028.

[11] J. Truksa, T. Gelbart, H. Peng, E. Beutler, B. Beutler, P. Lee, Suppression of the hepcidin-encoding gene Hamp permits iron overload in mice lacking both hemojuvelin and matriptase-2/TMPRSS6, Br. J. Haematol. 147 (2009) 571-581.

[12] K.E. Finberg, R.L. Whittlesey, M.D. Fleming, N.C. Andrews, Downregulation of $\mathrm{Bmp} / \mathrm{Smad}$ signaling by Tmprss6 is required for maintenance of systemic iron homeostasis, Blood 115 (2010) 3817-3826.

[13] H. Peng, J. Truksa, P. Lee, EPO-mediated reduction in Hamp expression in vivo corrects iron deficiency anaemia in TMPRSS6 deficiency, Br. J. Haematol. 151 (2010) 106-109.

[14] V. Niederkofler, R. Salie, S. Arber, Hemojuvelin is essential for dietary iron sensing, and its mutation leads to severe iron overload, J. Clin. Invest. 115 (2005) 2180-2186.

[15] A. Rodriguez Martinez, O. Niemelä, S. Parkkila, Hepatic and extrahepatic expression of the new iron regulatory protein hemojuvelin, Haematologica 89 (2004) 1441-1445.

[16] L. Lin, Y.P. Goldberg, T. Ganz, Competitive regulation of hepcidin mRNA by soluble and cell-associated hemojuvelin, Blood 106 (2005) 2884-2889.

[17] F.W. Huang, J.L. Pinkus, G.S. Pinkus, M.D. Fleming, N.C. Andrews, A mouse model of juvenile hemochromatosis, J. Clin. Invest. 115 (2005) 2187-2191.
[18] A.S. Zhang, A.P. West Jr., A.E. Wyman, P.J. Bjorkman, C.A. Enns, Interaction of hemojuvelin with neogenin results in iron accumulation in human embryonic kidney 293 cells, J. Biol. Chem. 280 (2005) 33885-33894.

[19] D. Kuninger, R. Kuns-Hashimoto, R. Kuzmickas, P. Rotwein, Complex biosynthesis of the muscle-enriched iron regulator RGMc, J. Cell Sci. 119 (2006) 3273-3283.

[20] L. Lin, E. Nemeth, J.B. Goodnough, D.R. Thapa, V. Gabayan, T. Ganz, Soluble hemojuvelin is released by proprotein convertase-mediated cleavage at a conserved polybasic RNRR site, Blood Cells Mol. Dis. 40 (2008) 122-131.

[21] A.S. Zhang, S.A. Anderson, K.R. Meyers, C. Hernandez, R.S. Eisenstein, C.A. Enns, Evidence that inhibition of hemojuvelin shedding in response to iron is mediated through neogenin, J. Biol. Chem. 282 (2007) 12547-12556.

[22] A.S. Zhang, F. Yang, J. Wang, H. Tsukamoto, C.A. Enns, Hemojuvelin-neogenin interaction is required for bone morphogenic protein-4-induced hepcidin expression, J. Biol. Chem. 284 (2009) 22580-22589.

[23] L. Silvestri, A. Pagani, C. Fazi, G. Gerardi, S. Levi, P. Arosio, C. Camaschella, Defective targeting of hemojuvelin to plasma membrane is a common pathogenetic mechanism in juvenile hemochromatosis, Blood 109 (2007) 4503-4510.

[24] A.S. Zhang, S.A. Anderson, J. Wang, F. Yang, K. DeMaster, R. Ahmed, C.P. Nizzi, R.S. Eisenstein, H. Tsukamoto, C.A. Enns, Suppression of hepatic hepcidin expression in response to acute iron deprivation is associated with an increase of matriptase-2 protein, Blood 117 (2011) 1687-1699.

[25] A.S. Zhang J. Gao, D.D. Koeberl, C.A. Enns, The role of hepatocyte hemojuvelin in the regulation of bone morphogenic protein- 6 and hepcidin expression in vivo, J. Biol. Chem. 285 (2010) 16416-16423. 